\title{
p120 catenin attenuates the angiotensin II-induced apoptosis of human umbilical vein endothelial cells by suppressing the mitochondrial pathway
}

\author{
YAN ZHANG $^{1 *}, \mathrm{CHENSHUANG} \mathrm{ZOU}^{2 *}$, SHUWEN YANG $^{1}$ and JING FU ${ }^{1}$ \\ ${ }^{1}$ Department of VIP Medical Service, ${ }^{2}$ Editorial Department of Chinese Journal \\ of Neuroimmunology and Neurology, Beijing Hospital, Beijing 100730, P.R. China
}

Received February 7, 2015; Accepted January 19, 2016

DOI: $10.3892 /$ ijmm.2016.2476

\begin{abstract}
Hypertension impairs the morphological and functional integrity of circulation. Previous research has shown that the loss of endothelial cells (ECs) is a common event in many cardiovascular diseases. p120 catenin (p120ctn) plays an important role in the regulation of inflammatory responses in ECs. However, the functional significance of p120ctn in angiotensin II (AngII)-induced apoptosis of human umbilical vein endothelial cells (HUVECs) had not previously received much scholarly attention. In the present study, using western blot analysis and RT-PCR, we found that AngII-induced cell apoptosis was correlated with a significant decrease in p120ctn expression. The effect of AngII on cell viability was measured by CCK-8 assay. Knockdown of p120ctn with small hairpin RNA (shRNA) increased AngII-induced apoptosis of HUVECs, as demonstrated by Annexin V/PI staining and flow cytometric analysis. Knockdown of p120ctn with shRNA also increased cytochrome $c$ release into the cytoplasm, and cleaved caspase- 3 and -9 protein expression. These were accompanied by a decrease in the $\mathrm{Bcl}-2 / \mathrm{Bax}$ ratio ( $\mathrm{Bcl}-2$ and $\mathrm{Bax}$ protein expression were measured by western blot analysis), and in mitochondrial membrane potential, as measured using JC-1. Overexpression of p120ctn with adenovirus produced opposite effects. In the present study, we demonstrated that p120ctn attenuated AngII-induced apoptosis of HUVECs through the mitochondria-dependent pathway, suggesting that p120ctn plays a critical role in protecting ECs against apoptosis during hypertension.
\end{abstract}

Correspondence to: Dr Yan Zhang, Department of VIP Medical Service, Ward D02, Beijing Hospital, 1 Dahua Road, Dongdan, Beijing 100730, P.R. China

E-mail: zhangyan_bjyy@126.com

*Contributed equally

Key words: p120 catenin, endothelial cells, apoptosis, angiotensin II

\section{Introduction}

Endothelial cells (ECs) play an essential role in maintaining endothelial homeostasis, which is involved in many vascular processes, including angiogenesis, vasoconstriction and vasodilation $(1,2)$. It has previously been reported that endothelial dysfunction underlies the pathogenesis of various cardiovascular diseases (3). EC injury, accompanied by an increase in the rate of apoptosis, has been noted as a common feature of endothelial dysfunction and in the pathogenesis of cardiovascular diseases such as hypertension (4). Angiotensin II (AngII), the main effector peptide of the reninangiotensin system (RAS), plays a pivotal role in regulating cardiovascular function via its specific receptors (5). Marked endothelial dysfunction induced by AngII has been observed in various cardiovascular diseases, including hypertension, atherosclerosis and myocardial infarction (6). AngII triggers excessive expression of reactive oxygen species (ROS), DNA damage, mitochondrial dysfunction and inflammation, as well as eventually inducing endothelial apoptosis (7-10). Notably, a previous study has demonstrated that blockade of AngII signaling by specific antagonists of the AngII type 1 receptor protects against hypertensive vascular injury, mainly through the inhibition of EC apoptosis (11). Although AngII has long been recognized as a mediator of endothelial apoptosis, the underlying mechanisms remain poorly understood.

p120 catenin (p120ctn) is a member of a subfamily of armadillo repeat proteins, and was originally identified as a substrate for Src kinase in 1989 (12). It has been proposed that it is an important component of adherens junctions (13). Previous studies have demonstrated that a number of proteins, including epithelial (E)-cadherin $(14,15)$, receptor tyrosine kinases (Src, Yes, Fer and Fyn) (16), transcription regulator Kaiso $(17,18)$, Rho GTPases (19), and Wnt signaling proteins such as glycogen synthase kinase-3 $\beta$ (GSK-3 $\beta$ ) (20), are physically or functionally regulated by $120 \mathrm{ctn}$, and this is indicative of the critical role played by p120ctn in the regulation of cell adhesion, proliferation, migration and cancer progression $(21,22)$. Interestingly, p120ctn deficiency in ECs exacerbates inflammatory responses in endotoxin-induced lung injury through activation of Tolllike receptor (TLR)4 signaling (23). Moreover, knockdown of p120ctn in ECs has been shown to activate the transcription 
of pro-inflammatory adhesion molecules and to trigger an inflammatory response (24), which is of significance during the process of EC apoptosis. However, the functional role of p120ctn in endothelial apoptosis had not been elucidated prior to this study. Thus, in the present study, we hypothesized that p120ctn plays an important role in the regulation of EC apoptosis. Our results demonstrated that p120ctn inhibits the apoptosis of human umbilical vein endothelial cells (HUVECs), which was induced by AngII through modulation of the mitochondria-dependent pathway. This suggests that p120ctn expression is a novel strategy which could be used to prevent EC apoptosis-related diseases, including hypertension.

\section{Materials and methods}

Cell culture. HUVECs were isolated upon collagenase treatment of human umbilical cord veins as previously described (5). After obtaining approval from the MedicalEthics Committee of Beijing Hospital and in accordance with the Declaration of Helsinki, human umbilical cords were collected following delivery (fullterm pregnancies) and after obtaining written informed consent from the mother. The cells were grown in M199 medium supplemented with $20 \%$ fetal calf serum, $50 \mu \mathrm{g} / \mathrm{ml}$ recombinant human endothelial cell growth factor, $25 \mathrm{U} / \mathrm{ml}$ heparin, $100 \mathrm{U} / \mathrm{ml}$ penicillin and $0.1 \mathrm{U} / \mathrm{ml}$ streptomycin (all from Gibco, Carlsbad, $\mathrm{CA}, \mathrm{USA}$ ) at $37^{\circ} \mathrm{C}$, in an atmosphere with $5 \% \mathrm{CO}_{2}$. HUVECs at passages 3-6 were used for all experiments.

Adenoviral infection. Human p120ctn adenovirus (Ad-p120ctn) was purchased from Cyagen Biosciences, Inc. (Santa Clara, CA, USA). The adenovirus expressing a small hairpin RNA (shRNA) sequence (GenBank accession no. NM_001331; 5'-GGACCUUACUGAAGUUAUUUU-3') targeting human p120ctn (Ad-shp1120ctn) was designed by and purchased from Sunbio Medical Biotechnology Co., Ltd., (Shanghai, China). A negative control (Lacz) was obtained from Clontech Laboratories (Mountain View, CA, USA) and untreated cells were used as controls. On the day prior to adenoviral infection, HUVECs were seeded in 6-well plates and reached $80 \%$ confluence. Ad-p120ctn or Ad-shp120ctn, at a different multiplicity of infection (MOI), was diluted in $2 \mathrm{ml} \mathrm{M} 199$ medium (Gibco) without serum and then added to the cells. After $3 \mathrm{~h}$, the cells were transferred into fresh culture medium and incubated for $48 \mathrm{~h}$ before analysis.

Cell viability assay. Cell viability was measured using a Cell Counting kit-8 (CCK-8; Dojindo Laboratories, Kumamoto, Japan) according to the manufacturer's instructions. Briefly, HUVECs were seeded in 96-well plates at a density of $2 \times 10^{3}$ cells/well. Following treatment with different concentrations of AngII (Sigma Chemical Co., St. Louis, MO, USA) for $24 \mathrm{~h}, 10 \mu \mathrm{l}$ CCK-8 was added to each well, followed by incubation at $37^{\circ} \mathrm{C}$ for an additional $3 \mathrm{~h}$. A colorimetric assay was performed using a Spectra Max 190 microplate reader (Molecular Devices LLC, Sunnyvale, CA, USA) and absorbance was measured at a wavelength of $450 \mathrm{~nm}$.

Cell apoptosis assay. Apoptosis of HUVECs was determined using an Accuri C6 flow cytometer and the FITC-Annexin V and propidium iodide (PI) double staining assay (both from
BD Biosciences, San Jose, CA, USA) according to the manufacturer's instructions. Briefly, the cells were digested with trypsin at a density of $1 \times 10^{6} / \mathrm{ml}$, washed with phosphate-buffered saline (PBS) three times, and then incubated with Annexin V and PI in the dark for $20 \mathrm{~min}$ at room temperature. The cells were counted with the flow cytometer, and the data were analyzed using CFlow Plus software (BD Biosciences).

Western blot analysis. Western blot analysis was performed as previously described (25). Briefly, HUVECs were lysed in RIPA lysis buffer (Beyotime Biotech, Jiangsu, China) supplemented with protease and phosphatase inhibitor cocktail (Sigma Chemical Co.). The cell lysates were separated by 8-12\% sodium dodecyl sulfate-polyacrylamide gel electrophoresis (SDS-PAGE), and then transferred to polyvinylidene fluoride membranes (Millipore Corp., Billerica, MA, USA). The membranes were blocked in 5\% non-fat milk for $1 \mathrm{~h}$ at room temperature, and then incubated with the following primary antibodies: p120ctn (1:500; 612536; BD Biosciences); Bcl-2 (\#4223), Bax (\#5023), cytochrome c (\#11940), caspase-3 (\#9669), and caspase-9 (1:2,000; \#9509; all from Cell Signaling Technology, Danvers, MA, USA); Cox-IV (SC-69360), GAPDH (SC-365062), and $\beta$-actin (1:1,000; SC-8430; all from Santa Cruz Biotechnology, Inc., Santa Cruz, CA, USA). After washing, secondary antibodies, namely HRP-conjugated anti-rabbit (\#7074) or anti-mouse (1:1,000; \#7076; both from Cell Signaling Technology), were incubated for $1 \mathrm{~h}$ at room temperature, and the membranes were then visualized using a chemiluminescence kit (Thermo Fisher Scientific, Inc., Rockford, IL, USA).

Reverse transcription-polymerase chain reaction (RT-PCR) analysis of pl20ctn expression. Total RNA from HUVECs was isolated using TRIzol reagent (Invitrogen, Carlsbad, CA, USA) following the manufacturer's instructions. The purity and concentration of RNA were determined by nucleic acid quantitative instrument (Qubit 2.0; Invitrogen). One microgram of total RNA was used to performed the RT-PCR with a One Step RT-PCR kit (Qiagen, Inc., Valencia, CA, USA). The reaction conditions were as follows: $95^{\circ} \mathrm{C}$ for $30 \mathrm{sec}, 60^{\circ} \mathrm{C}$ for $45 \mathrm{sec}, 72^{\circ} \mathrm{C}$ for $60 \mathrm{sec}$ ( 32 cycles). The specific primers for p120ctn and GAPDH were synthesized by Invitrogen as follows: p120ctn, 5'-TACGCTCTCTCCTTCCTGCT-3' and 5'-AGACATGGCTCCCTCAGGAT-3'; and GAPDH, 5'-GGG CACGAAGGCTCATCATT-3' and 5'-AGAAGGCTGGGG CTCATTTG-3'. The amplified products were electrophoresed on $1 \%$ agarose gels and the bands were analyzed using ImageJ software (NIH, Bethesda, MD, USA).

Preparation of mitochondrial fractions. Following treatment, HUVECs were harvested and suspended in $4 \mathrm{ml}$ mitochondrial isolation buffer $(200 \mathrm{mM}$ mannitol, $75 \mathrm{mM}$ sucrose, $1 \mathrm{mM}$ EDTA, $1 \%$ cocktail protease inhibitor, $5 \mathrm{mM}$ Tris/ $\mathrm{HCl} \mathrm{pH} \mathrm{7.4).} \mathrm{The} \mathrm{homogenates} \mathrm{were} \mathrm{centrifuged} \mathrm{at} \mathrm{2,500} \mathrm{rpm}$ twice for $5 \mathrm{~min}$ at $4^{\circ} \mathrm{C}$, and the supernatant contained the cytosolic proteins. The pellet was resuspended in mitochondrial isolation buffer and placed on top of sucrose density gradient buffer (1.0, 1.2 and 1.5 M sucrose). The samples were centrifuged at $25,000 \mathrm{rpm}$ for $30 \mathrm{~min}$ at $4^{\circ} \mathrm{C}$ on a Sorvall TST60.4 rotor (Beckman Coulter, Krefeld, Germany). Mitochondrial- 

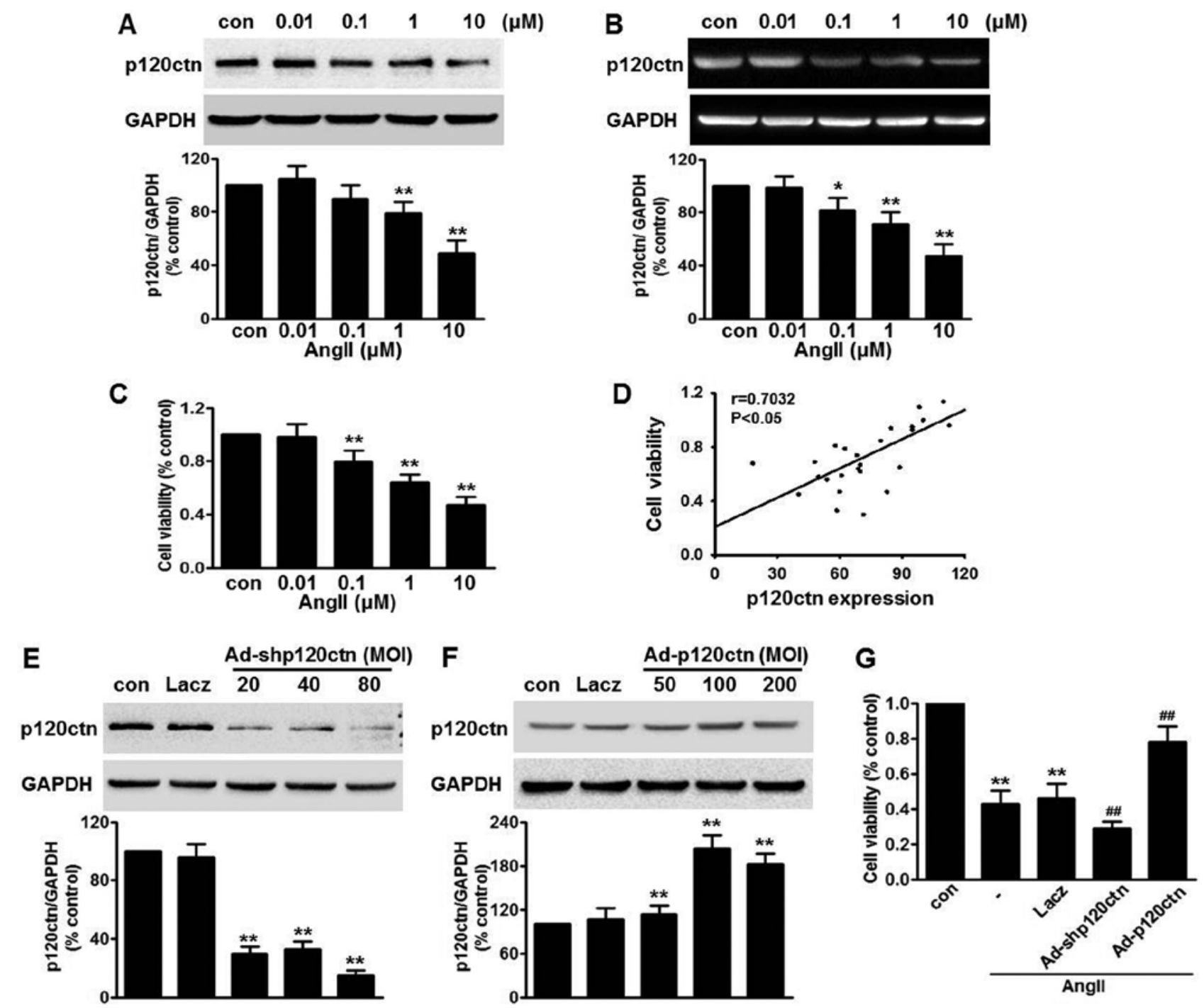

Figure 1. Effect of p120 catenin (p120ctn) and angiotensin II (AngII) on human umbilical vein endothelial cells (HUVECs). (A-D) HUVECs were treated with AngII at different concentrations $(0.01,0.1,1$ and $10 \mu \mathrm{M})$ for $24 \mathrm{~h}$. p120ctn expression was detected by (A) western blot analysis and (B) RT-PCR. (C) Cell viability was determined by CCK-8. (D) Correlation between cell viability and p120ctn expression was analyzed. (E-F) HUVECs were infected with a small hairpin RNA (shRNA) targeting (E) p120ctn [multiplicity of infection (MOI) of 20, 40 and 80] or (F) p120ctn cDNA (MOI of 50, 100 and 200) for $48 \mathrm{~h}$, and infection efficiency was determined by western blot analysis. (G) The cells were infected with Lacz (MOI of 100), Ad-shp120ctn (MOI of 80), or Ad-120ctn (MOI of 100) for $48 \mathrm{~h}$, followed by incubation with AngII $(10 \mu \mathrm{M})$ for $24 \mathrm{~h}$. Cell viability was evaluated by a CCK-8. All data are presented as the means \pm SEM. ${ }^{*} \mathrm{P}<0.05$ and ${ }^{* *} \mathrm{P}<0.01$ vs. control; ${ }^{\# \#} \mathrm{P}<0.01$ vs. AngII alone, $\mathrm{n}=6$.

enriched fractions were collected at $1.2 / 1.5 \mathrm{M}$ sucrose interphases and analyzed by western blot analysis.

Examination of mitochondrial membrane potential (MMP). In the present study, 5,5',6,6'-Tetrachloro-1,1',3,3'-tetraethylbenza-midazolocarbocyanin iodide (JC-1 dye; Molecular Probes $^{\mathrm{TM}}$, Invitrogen, Carlsbad, CA, USA) was used to measure MMP. JC-1 is a cationic dye that exhibits potential-dependent accumulation in mitochondria, which is indicated by red fluorescence (excitation, $550 \mathrm{~nm}$; emission, $600 \mathrm{~nm}$ ) in viable cells. During apoptosis, JC-1 exists in the cytoplasm as a monomer, indicated by green fluorescence (excitation, $485 \mathrm{~nm}$; emission, $535 \mathrm{~nm}$ ). Consequently, the red/green fluorescence intensity ratio indicates changes to MMP.

Statistical analysis. In the present study, all data are expressed as the means \pm SEM, and $\mathrm{n}$ indicates the number of independent experiments. A one-way analysis of variance (ANOVA) followed by Tukey's multiple comparison post-hoc test was used in order to analyze the differences of multiple groups. A P-value $<0.05$ was considered to indicate a statistically significant difference in all statistical tests. All statistical analyses were performed using SPSS version 15 statistical software (SPSS Inc., Chicago, IL, USA).

\section{Results}

Effects of AngII and p120ctn on HUVEC viability. We first investigated the effect of AngII on p120ctn expression in HUVECs. Western blot analysis revealed that p120ctn protein expression decreased in a concentration-dependent manner following treatment with AngII for $24 \mathrm{~h}$. AngII at a concentration of $10 \mu \mathrm{M}$ significantly decreased p120ctn protein expression to $54.7 \pm 6.9 \%$ compared with the control group (Fig. 1A). RT-PCR 

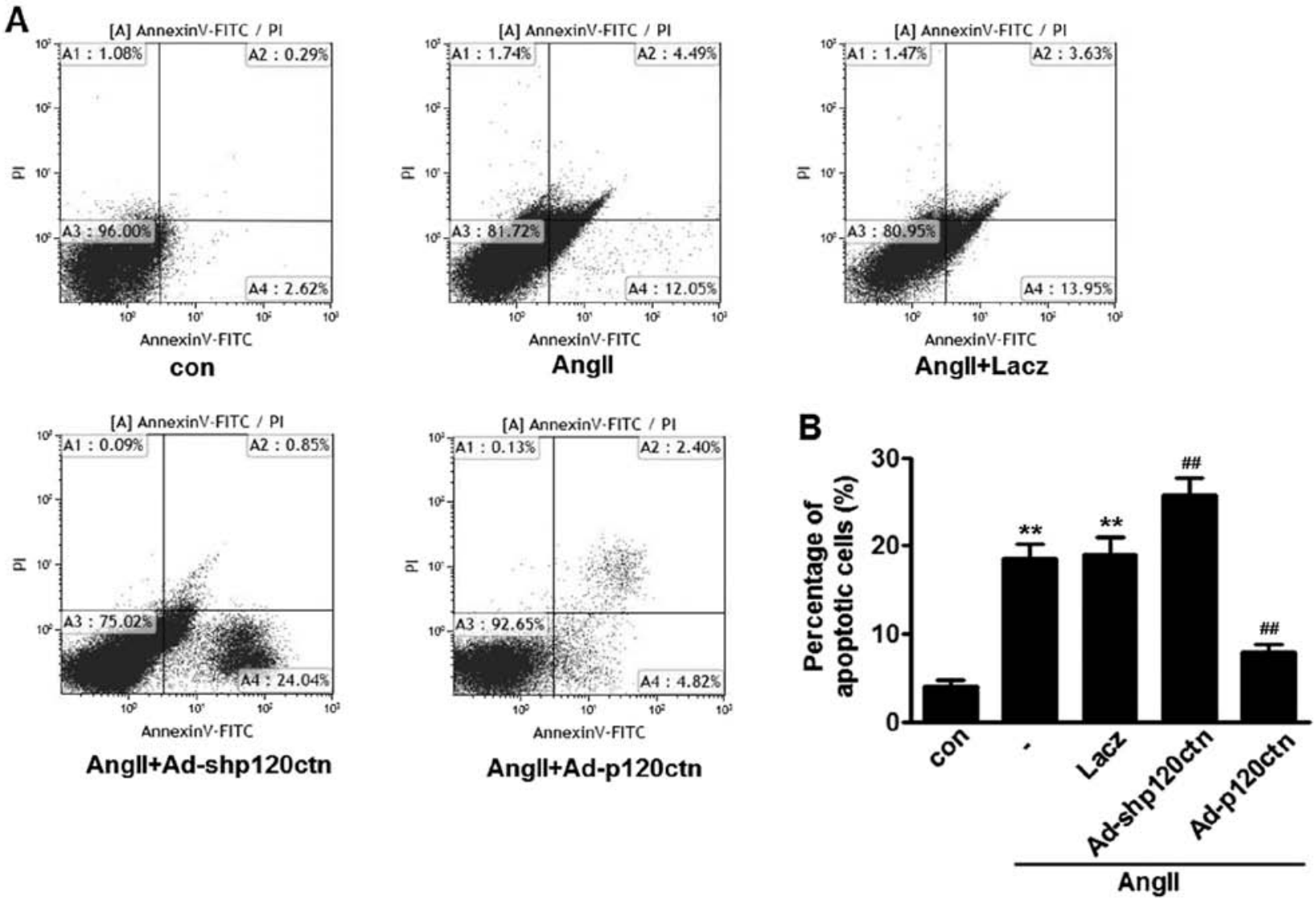

Figure 2. p120 catenin (p120ctn) attenuates angiotensin II (AngII)-induced apoptosis of human umbilical vein endothelial cells (HUVECs). (A) HUVECs were infected with Lacz [multiplicity of infection (MOI) of 100], Ad-shp120ctn (MOI of 80), or Ad-120ctn (MOI of 100) for $48 \mathrm{~h}$, and then treated with AngII $(10 \mu \mathrm{M})$ for another $24 \mathrm{~h}$. Apoptosis of HUVECs was determined by Annexin V/PI staining followed by flow cytometric analysis. (B) Quantitative analysis of the percentage of apoptotic cells. ${ }^{* *} \mathrm{P}<0.01$ vs. control; ${ }^{\# \prime} \mathrm{P}<0.01$ vs. AngII alone, $\mathrm{n}=6$.

revealed a similar trend in p120ctn mRNA expression after challenge with AngII (Fig. 1B), indicating that AngII decreased p120ctn expression, at least in part, through a post-transcriptional mechanism. The effect of AngII on cell viability was measured by CCK- 8 assay. AngII decreased cell viability in a concentration-dependent manner (Fig. 1C). Compared with the control, at concentrations of $0.1,1$ and $10 \mu \mathrm{M}$, cell viability was reduced to $78.2 \pm 9.3,63.5 \pm 7.8$ and $45.2 \pm 3.3 \%$, respectively. We found p120ctn protein expression to be positively correlated with cell viability after AngII treatment (Fig. 1D), suggesting that p120ctn expression is involved in AngII-induced injury to HUVECs. In order to verify this observation, we used adenoviruses bearing shRNA or p120ctn cDNA to knock down or overexpress p120ctn. Efficiency of infection was detected by western blot analysis. Ad-shp120ctn at an MOI of 80 decreased endogenous p120ctn expression $>80 \%$ compared with the Lacz group (Fig. 1E). Ad-p120ctn significantly increased p120ctn expression, and the peak increase was observed at an MOI of 100, while Lacz produced no marked effect (Fig. 1F). Thus, we selected Ad-shp120ctn at an MOI of 80 and Ad-p120ctn at an MOI of 100 for use in the following experiments. The CCK-8 assay revealed that neither p120ctn knockdown nor overexpression exerted a significant effect on cell viability (data not shown). However, Ad-shp120ctn further augmented AngII-induced injury to HUVECs, reducing the cell viability to $36.3 \pm 2.7 \%$ of the control group. As expected, overexpression of p120ctn markedly inhibited the decrease in cell viability caused by AngII (Fig. 1G).

p120ctn attenuates AngII-induced apoptosis of HUVECs. To determine whether decreased p120ctn is involved in AngII-induced apoptosis of HUVECs, quantitative analysis of apoptosis by flow cytometric analysis was performed. The Annexin V-FITC/PI data revealed that incubation with AngII for $24 \mathrm{~h}$ increased the apoptotic rate to $17.2 \pm 1.9 \%$ in HUVECs. p120ctn-specific shRNA further increased the apoptotic cell population to $26.1 \pm 2.7 \%$, whereas p120ctn overexpression markedly abrogated AngII-induced apoptosis of HUVECs. Lacz infection did not alter the increase in apoptotic cells caused by transfection with AngII (Fig. 2). These results suggest that p120ctn exerts no effect on apoptosis of HUVECs at basal levels; however, p120ctn attenuates AngII-induced apoptosis.

Effect of p120ctn on AngII-induced expression of the apoptosisassociated proteins, Bcl-2 and Bax. In order to investigate the mechanism by which p120ctn regulates AngII-induced apoptosis of HUVECs, western blot analysis was performed to examine the expression of Bcl-2 and Bax, two Bcl-2 family members which are known to be important in the regulation of apoptosis (27,32) (Fig. 3A). Low levels of Bcl-2 and high levels of Bax expression were detected following AngII treat- 
A

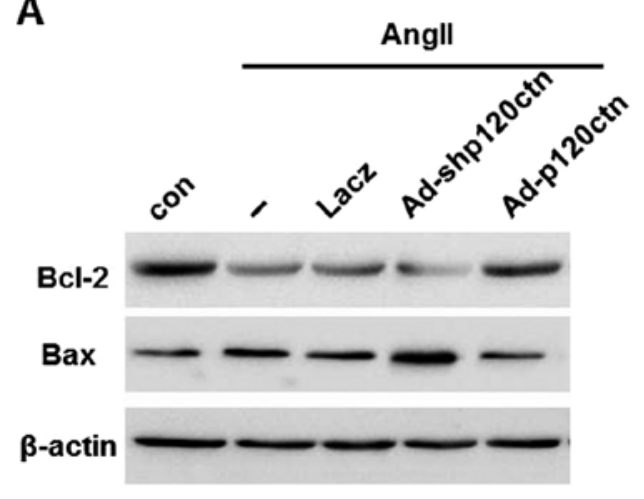

B

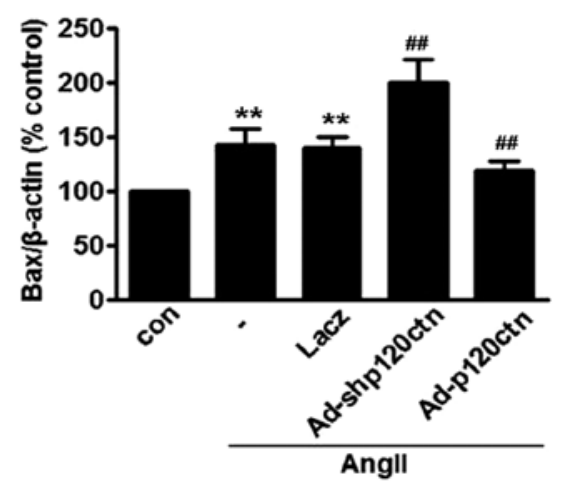

C

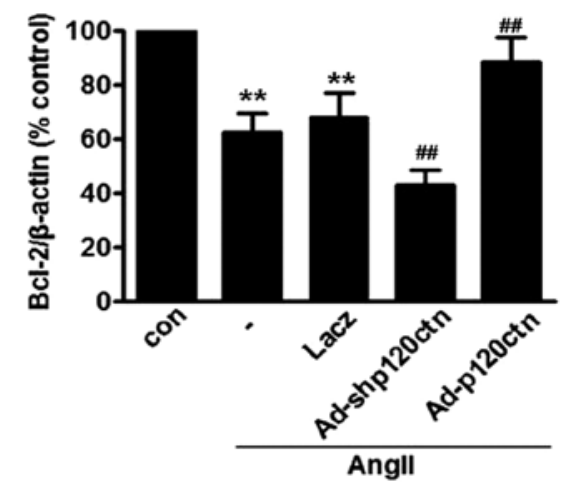

D

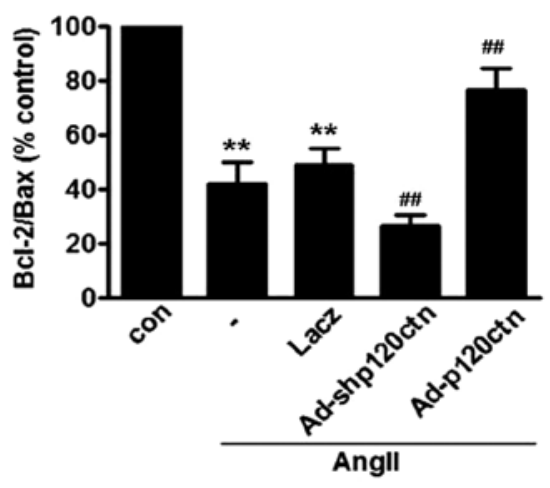

Figure 3. Effect of p120 catenin (p120ctn) on angiotensin II (AngII)-induced expression of Bcl-2 and Bax. (A-D) After infection, Bcl-2 and Bax expression were examined by western blot analysis. (A) Representative western blot analysis images are shown. Densitometric analysis of (B) Bcl-2, (C) Bax and (D) Bcl-2/Bax ratio. ${ }^{* *} \mathrm{P}<0.01$ vs. control; ${ }^{\# \prime} \mathrm{P}<0.01$ vs. AngII alone, $\mathrm{n}=6$.

ment for $24 \mathrm{~h}$. Furthermore, the ratio of Bcl-2 to Bax, which appears to determine cell fate (survival or death), was markedly reduced by AngII treatment. As expected, knockdown of p120ctn further augmented the effect of AngII on Bcl-2 and Bax expression, and the Bcl-2/Bax ratio. Moreover, upregulation of p120ctn reversed the AngII-decreased Bcl-2/Bax ratio, due to an increase in Bcl-2 expression and a decrease in Bax expression (Fig. 3B-D).

Effect of p120ctn on AngII-induced MMP depolarization and cytochrome $c$ release. A previous study has shown that MMP plays an essential role in the AngII-induced apoptosis of HUVECs (26). Consistent with this study, we found that AngII induced loss of MMP in HUVECs. Confocal microscopy revealed that AngII decreased red JC-1 fluorescence and increased green JC-1 fluorescence, respectively, resulting in a decline in the red/green fluorescence ratio (Fig. 4A and B). We further explored whether p120ctn attenuated AngII-induced apoptosis of HUVECs by regulating MMP. We noted that p120ctn knockdown markedly enhanced the AngII-induced decrease in MMP, and in the Ad-p120ctn-treated cells this decrease was reduced (Fig. 4A and B).

Impairment of the mitochondrial pathway has previously been suggested to induce the release of cytochrome $c$ from mitochondrial into the cytoplasm (27). Following incubation with AngII for $24 \mathrm{~h}$, the translocation of cytochrome $c$ from mitochondria into the cytoplasm was significantly increased, suggesting that the increase of cytochrome $c$ in cytoplasm may be involved in apoptosis induced by AngII. The increased translocation of cytochrome $c$ into the cytoplasm was further enhanced in Ad-shp120ctn-infected cells. However, we found that overexpression of p120ctn markedly impaired AngII-induced translocation of cytochrome $c$ (Fig. 4C and D).

Collectively, these data indicate that the stabilization of MMP and the impairment of cytochrome $c$ translocation underlie, at least partially, the protective effect of p120ctn on the AngII-induced apoptosis of HUVECs.

p120ctn abolishes AngII-induced activation of caspase-3 and -9 . Cytochrome $c$ released from mitochondria cleave and activate downstream apoptosis-associated proteins, such as caspase- 3 and caspase- 9 , which in turn initiate mitochondriadependent apoptosis (28). Our results showed that AngII increased cleaved caspase- 3 and caspase-9. The activation of caspase-3 and caspase-9 was further enhanced in HUVECs transfected with Ad-shp120ctn, whereas overexpression of p120ctn almost abolished the caspase activation induced by AngII. The adenovirus bearing Lacz did not markedly alter this activation (Fig. 5).

\section{Discussion}

p120ctn is best known for its role in the inflammatory responses; previous studies of conditional targeting of p120ctn in knockout mice have noted increased inflammatory cell infiltration and pro-inflammatory cytokines, resulting from activation of the nuclear factor- $\kappa \mathrm{B}(\mathrm{NF}-\kappa \mathrm{B})$ pathway $(25,29)$. Deficiency of p120ctn markedly potentiates lipopolysac- 

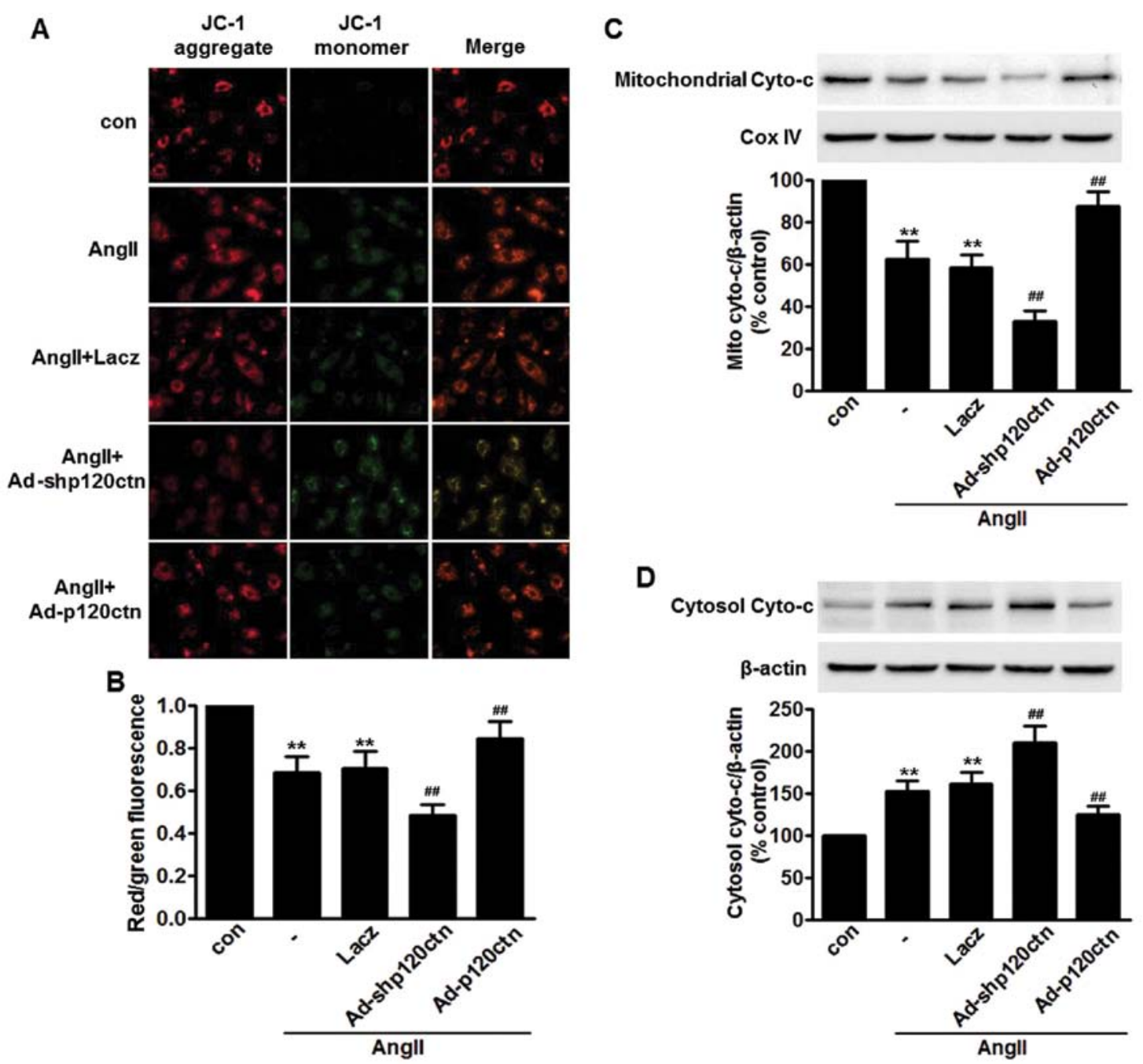

Figure 4. Protective effect of p120 catenin (p120ctn) on angiotensin II (AngII)-induced apoptosis of human umbilical vein endothelial cells (HUVECs) through the mitochondria pathway. (A) HUVECs were infected with Lacz [multiplicity of infection (MOI) of 100], Ad-shp120ctn (MOI of 80), or Ad-120ctn (MOI of $100)$ for $48 \mathrm{~h}$, and then treated with AngII $(10 \mu \mathrm{M})$ for another $24 \mathrm{~h}$. Mitochondrial membrane potential was measured using JC-1 staining. Representative images of JC-1 derived fluorescence in HUVECs. The JC-1 aggregate image shows red fluorescence; the JC-1 monomer image shows green fluorescence; the merged image combines the red and green images. (B) Quantitative analysis of the ratio of red/green fluorescence. (C and D) The cells were treated as shown in (A); cytochrome $c$ protein expression in the (C) mitochondria and (D) cytosol was determined by western blot analysis. Cox IV was used as a loading control of mitochondrial protein. ${ }^{* *} \mathrm{P}<0.01$ vs. control; ${ }^{\# /} \mathrm{P}<0.01$ vs. AngII alone, $\mathrm{n}=6$.
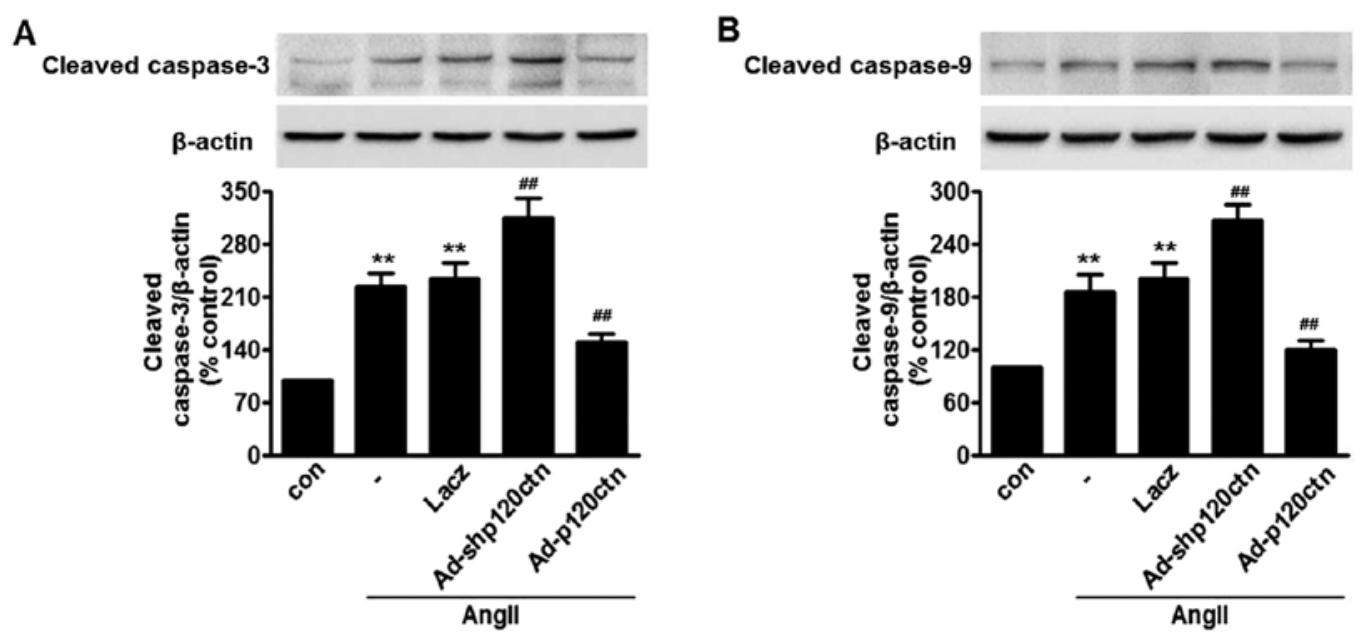

Figure 5. Effect of p120 catenin (p120ctn) on angiotensin II (AngII)-induced caspase activation. (A and B) Human umbilical vein endothelial cells (HUVECs) were treated with Lacz [multiplicity of infection (MOI) of 100], Ad-shp120ctn (MOI of 80), or Ad-120ctn (MOI of 100) for $48 \mathrm{~h}$. AngII (10 $\mu \mathrm{M}$ ) was then added into the culture medium for a further $24 \mathrm{~h}$. Protein levels of (A) caspase-3 and (B) caspase- 9 were measured by western blot analysis. ${ }^{* *} \mathrm{P}<0.01 \mathrm{vs}$. control; ${ }^{\# \#} \mathrm{P}<0.01$ vs. AngII alone, $\mathrm{n}=6$. 
charide (LPS)-induced inflammation in human brain microvascular endothelial cells and human bronchial epithelial cells $(30,31)$. Notably, p120ctn also functions as an endogenous anti-inflammatory molecule in ECs. While the role of p120ctn with respect to endothelial inflammation has been previously described, its role in inflammation-associated apoptosis had not been explored in detail. In the present study, we investigated the association between p120ctn and AngII-induced apoptosis of HUVECs. We have reported, for the first time to the best of our knowledge, that endogenous p120ctn expression was decreased following AngII treatment. This reduction was accompanied by a decrease in cell viability, in the same concentration-dependent manner. Moreover, knockdown of p120ctn augmented AngIIinduced apoptosis of HUVECs and mitochondrial dysfunction. By contrast, overexpression of p120ctn produced the opposite effects. Thus, the data from the present study indicate that p120ctn plays a protective role in EC apoptosis.

Apoptosis of vascular ECs has been shown to play a critical role in endothelial dysfunction; importantly, EC apoptosis is an initiating, early mechanism for hypertension, leading directly to microvascular obliteration (33). It has been proved that the main effector of the renin-angiotensin-aldosterone system, AngII, is involved in the pathogenesis of hypertension and results in the apoptosis of ECs $(34,35)$. We suggest that inhibition of AngII-induced apoptosis of ECs is an important strategy to prevent cardiovascular diseases, including hypertension. In the present study, we found that AngII treatment significantly decreased cell viability and induced cell apoptosis, supporting the theory that AngII contributes to endothelial dysfunction, as noted in previous research (26). Of note, our study has revealed, for the first time to the best of our knowledge, that p120ctn expression was decreased after challenge with AngII. AngIIinduced apoptosis of HUVECs was found to be accompanied by a decrease in p120ctn expression, indicating that p120ctn may be a critical regulator of EC apoptosis in AngII-related hypertension. AngII-induced apoptosis of HUVECs was almost abolished after infection with p120ctn adenovirus, whereas apoptosis was markedly enhanced in HUVECs transfected with p120ctn shRNA. These data further confirm the essential role which p120ctn plays in EC apoptosis.

We next explored the mechanism linking p120ctn and AngII-induced apoptosis of ECs. It is known that the mitochondrial pathway is a major signaling pathway involved in the apoptotic process; changes in MMP usually correlate with alterations in Bcl-2 and Bax expression (36). These two proteins are crucial to the apoptotic machinery and a wide array of diverse upstream survival signals, thus determining the fate of the cells $(32,37)$. Upon apoptotic stimulation, the balance of Bcl-2 and Bax is broken, the increased level of Bax in mitochondria induces MMP depolarization, which is followed by cytochrome $c$ release from mitochondria into the cytosol, which combines with caspase- 9 to form a complex, and activates caspase-3, triggering apoptosis $(27,28)$. Consistent with a previous study (26), AngII treatment of HUVECs markedly decreased Bcl-2 and increased Bax expression, concomitantly with the loss of MMP and the increase of caspase-3 and -9 , suggesting that the mitochondrial pathway is involved in AngII-induced apoptosis. The results of the present study have demonstrated that p120ctn shRNA enhanced the decrease in the $\mathrm{Bcl}-2 / \mathrm{Bax}$ ratio, the depolarization of MMP, the increase in cytochrome $c$ release into the cytoplasm and the activation of capase-3 and -9 induced by AngII. However, overexpression of p120ctn produced the opposite effects. These results indicate that the effect of p120ctn on apoptosis in HUVECs is mitochondria-dependent.

In conclusion, the present study provides evidence that p120ctn attenuates AngII-induced apoptosis of ECs. The anti-apoptotic effects of p120ctn are mainly due to inhibition of the mitochondria-dependent pathway. These findings assist us in gaining a better understanding of the role of p120ctn in apoptosis and also provide a novel strategy for the prevention of endothelial dysfunction in cardiovascular diseases, such as hypertension.

\section{Acknowledgments}

This study was supported by the Natural Science Foundation (no. 51272181).

\section{References}

1. Lee R, Channon KM and Antoniades C: Therapeutic strategies targeting endothelial function in humans: clinical implications. Curr Vasc Pharmacol 10: 77-93, 2012.

2. Shi H, Sheng B, Zhang F, Wu C, Zhang R, Zhu J, Xu K, Kuang Y, Jameson SC, Lin Z, et al: Kruppel-like factor 2 protects against ischemic stroke by regulating endothelial blood brain barrier function. Am J Physiol Heart Circ Physiol 304: H796-H805, 2013.

3. Wada K, Gerbaudo VH and Spector M: Effects of PDGF-BB and OP-1 on mesenchymal stem cells in a porous mineral block. Int J Periodontics Restorative Dent 33: e72-e78, 2013.

4. Xie F, Cai W, Liu Y, Li Y, Du B, Feng L and Qiu L: Vaccarin attenuates the human EA.hy926 endothelial cell oxidative stress injury through inhibition of Notch signaling. Int J Mol Med 35: 135-142, 2015.

5. Wang C, He Y, Yang M, Sun H, Zhang S and Wang C: Safflor yellow B suppresses angiotensin II-mediated human umbilical vein cell injury via regulation of $\mathrm{Bcl}-2 / \mathrm{p} 22$ (phox) expression. Toxicol Appl Pharmacol 273: 59-67, 2013.

6. Dimmeler S, Rippmann V, Weiland U, Haendeler J and Zeiher AM: Angiotensin II induces apoptosis of human endothelial cells. Protective effect of nitric oxide. Circ Res 81: 970-976, 1997.

7. Mendell JT and Olson EN: MicroRNAs in stress signaling and human disease. Cell 148: 1172-1187, 2012.

8. Caporali A and Emanueli C: MicroRNA regulation in angiogenesis. Vascul Pharmacol 55: 79-86, 2011.

9. Wang J, Sun P, Bao Y, Dou B, Song D and Li Y: Vitamin E renders protection to $\mathrm{PC} 12$ cells against oxidative damage and apoptosis induced by single-walled carbon nanotubes. Toxicol In Vitro 26: 32-41, 2012.

10. Paravicini TM and Touyz RM: Redox signaling in hypertension. Cardiovasc Res 71: 247-258, 2006.

11. Sata M and Fukuda D: Crucial role of renin-angiotensin system in the pathogenesis of atherosclerosis. J Med Invest 57: 12-25, 2010.

12. Reynolds AB, Roesel DJ, Kanner SB and Parsons JT: Transformation-specific tyrosine phosphorylation of a novel cellular protein in chicken cells expressing oncogenic variants of the avian cellular src gene. Mol Cell Biol 9: 629-638, 1989.

13. Wang M, Li N, Li J, Ma Y, Li D, Qin L, Wang X and Wu R: Involvement of p120 in LPS-induced NF-kappaB activation and IL- 8 production in human bronchial epithelial cells. Toxicol Lett 195: 75-81, 2010.

14. Reynolds AB, Daniel J, McCrea PD, Wheelock MJ, Wu J and Zhang Z: Identification of a new catenin: the tyrosine kinase substrate p120cas associates with E-cadherin complexes. Mol Cell Biol 14: 8333-8342, 1994.

15. Shibamoto S, Hayakawa M, Takeuchi K, Hori T, Miyazawa K, Kitamura N, Johnson KR, Wheelock MJ, Matsuyoshi N, Takeichi $\mathrm{M}$ and Fumiaki Ito: Association of p120, a tyrosine kinase substrate, with E-cadherin/catenin complexes. J Cell Biol 128: 949-957, 1995. 
16. Piedra J, Miravet S, Castaño J, Pálmer HG, Heisterkamp N García de Herreros A and Duñach M: p120 Catenin-associated Fer and Fyn tyrosine kinases regulate beta-catenin Tyr-142 phosphorylation and beta-catenin-alpha-catenin Interaction. Mol Cell Biol 23: 2287-2297, 2003.

17. Kim SW, Park JI, Spring CM, Sater AK, Ji H, Otchere AA, Daniel JM and McCrea PD: Non-canonical Wnt signals are modulated by the Kaiso transcriptional repressor and p120-catenin. Nat Cell Biol 6: 1212-1220, 2004.

18. Prokhortchouk A, Hendrich B, Jørgensen H, Ruzov A, Wilm M, Georgiev G, Bird A and Prokhortchouk E: The p120 catenin partner Kaiso is a DNA methylation-dependent transcriptional repressor. Genes Dev 15: 1613-1618, 2001.

19. Anastasiadis PZ, Moon SY, Thoreson MA, Mariner DJ, Crawford HC, Zheng Y and Reynolds AB: Inhibition of RhoA by p120 catenin. Nat Cell Biol 2: 637-644, 2000.

20. Hong JY, Park JI, Cho K, Gu D, Ji H, Artandi SE and McCrea PD: Shared molecular mechanisms regulate multiple catenin proteins: canonical Wnt signals and components modulate p120catenin isoform-1 and additional p120 subfamily members. J Cell Sci 123: 4351-4365, 2010

21. Reynolds AB: p120-catenin: past and present. Biochim Biophys Acta 1773: 2-7, 2007.

22. Reynolds $A B$ and Roczniak-Ferguson A: Emerging roles for p120-catenin in cell adhesion and cancer. Oncogene 23: 7947-7956, 2004.

23. Wang YL, Malik AB, Sun Y, Hu S, Reynolds AB, Minshall RD and $\mathrm{Hu}$ G: Innate immune function of the adherens junction protein p120-catenin in endothelial response to endotoxin. J Immunol 186: 3180-3187, 2011.

24. O'Donnell JJ III, Zhuge Y, Holian O, Cheng F, Thomas LL, Forsyth CB and Lum H: Loss of p120 catenin upregulates transcription of pro-inflammatory adhesion molecules in human endothelial cells. Microvasc Res 82: 105-112, 2011.

25. Perez-Moreno M, Davis MA, Wong E, Pasolli HA, Reynolds AB and Fuchs E: p120-catenin mediates inflammatory responses in the skin. Cell 124: 631-644, 2006.

26. Liu J, Zhang FF, Li L, Yang J, Liu J, Guan YY and Du YH: ClC-3 deficiency prevents apoptosis induced by angiotensin II in endothelial progenitor cells via inhibition of NADPH oxidase. Apoptosis 18: 1262-1273, 2013.

27. Ott M, Gogvadze V, Orrenius S and Zhivotovsky B: Mitochondria, oxidative stress and cell death. Apoptosis 12: 913-922, 2007.
28. Mignotte B and Vayssiere JL: Mitochondria and apoptosis. Eur J Biochem 252: 1-15, 1998.

29. Perez-Moreno M, Song W, Pasolli HA, Williams SE and Fuchs E: Loss of p120 catenin and links to mitotic alterations, inflammation, and skin cancer. Proc Natl Acad Sci USA 105: 15399-15404, 2008.

30. Liu N, Li AL, Zhou XP, Chen Q and Cao W: P120 catenin attenuates lipopolysaccharide-induced blood-brain barrier dysfunction and inflammatory responses in human brain microvascular endothelial cells. Int J Clin Exp Pathol 8: 4204-4212, 2015.

31. Qin L, Qin S, Zhang Y, Zhang C, Ma H, Li N, Liu L, Wang X and Wu R: p120 modulates LPS-induced NF- $\kappa$ B activation partially through RhoA in bronchial epithelial cells. Biomed Res Int 2014: 932340, 2014.

32. Ye QF, Zhang YC, Peng XQ, Long Z, Ming YZ and HeLY: Silencing Notch-1 induces apoptosis and increases the chemosensitivity of prostate cancer cells to docetaxel through Bcl-2 and Bax. Oncol Lett 3: 879-884, 2012.

33. Teichert-Kuliszewska K, Tsoporis JN, Desjardins JF, Yin J, Wang L, Kuebler WM and Parker TG: Absence of the calciumbinding protein, S100A1, confers pulmonary hypertension in mice associated with endothelial dysfunction and apoptosis. Cardiovasc Res 105: 8-19, 2015.

34. Efrati S, Berman S, Goldfinger N, Erez N, Averbukh Z, Golik A, Rotter V and Weissgarten J: Enhanced angiotensin II production by renal mesangium is responsible for apoptosis/proliferation of endothelial and epithelial cells in a model of malignant hypertension. J Hypertens 25: 1041-1052, 2007.

35. Rojas E, Rodríguez-Molina D, Bolli P, Israili ZH, Faría J, Fidilio E, Bermúdez V and Velasco M: The role of adiponectin in endothelial dysfunction and hypertension. Curr Hypertens Rep 16: 463, 2014

36. Misiti F, Orsini F, Clementi ME, Lattanzi W, Giardina B and Michetti F: Mitochondrial oxygen consumption inhibition importance for TMT-dependent cell death in undifferentiated PC12 cells. Neurochem Int 52: 1092-1099, 2008.

37. Reagan-Shaw S, Nihal M, Ahsan H, Mukhtar H and Ahmad N: Combination of vitamin $\mathrm{E}$ and selenium causes an induction of apoptosis of human prostate cancer cells by enhancing $\mathrm{Bax} / \mathrm{Bcl}-2$ ratio. Prostate 68: 1624-1634, 2008. 